IDDF2019-ABS-0315 FAMILIAL ADENOMATOUS POLYPOSIS AND ITS ASSOCIATIONS : EXPERIENCE FROM TERTIARY REFERRAL CENTRE IN INDIA

Pooja Keshan*. LTMMC and General hospital, Mumbai, India

\subsection{6/gutjnl-2019-IDDFabstracts.72}

Background Familial Adenomatous Polyps (FAP) is an autosomal dominant rare condition characterised by more than 100 adenomatous polyps with a high risk of colonic carcinoma. It can be also associated with extracolonic neoplasms. Herein we present with 4 cases of FAP associated with upper and lower GI tumours within a period of 6 months.

Our aim was to study the association of FAP with various neoplasms

Methods Indian data on FAP and its variants is scarce and is limited to case reports due to its low incidence. We report our experience of 4 cases of FAP in a duration of 6 months (July 2018- December 2018) with its associated manifestations. Results The first patient was a 41-year-old female who presented with bleeding PR, mucus in stools and abdominal pain. She was a known case of FAP with restorative proctocolectomy and ileoanal anastomosis done. After 10 years of the initial surgery ulceroproliferative periampullary growth was identified which was diagnosed as moderately differentiated adenocarcinoma. Another case was a 58-year-old female who presented with complaints of bleeding PR with palpable rectal mass. She was diagnosed as attenuated FAP with tubular and tubulo-villous adenomas on histology. There was a rectal growth showing features of synchronous adenocarcinoma. Remaining 2 cases of FAP (46-year male, 13-year female) presented with bleeding PR and abdominal pain. Follow up upper gastrointestinal endoscopy revealed fundic gland polyps in the stomach. One of which also showed the presence of gastric adenoma in the antrum.

Conclusions FAP can present with a variety of intestinal and extraintestinal manifestations. This case series highlights the need for upper gastrointestinal scopy in patients with FAP. It also focuses on the need for increased awareness of the syndrome and its variants while treating patients with polyposis related conditions.

\section{IDDF2019-ABS-0316 LONG NON-CODING RNA CRCAL-2 PROMOTES GASTRIC CANCER METASTASIS BY ACTIVATING WNT/BETA-CATENIN PATHWAY VIA STABILIZING THE NUCLEAR TRANSPORT PROTEIN RAN}

${ }^{1}$ Qi Meng ${ }^{*},{ }^{1}$ Yun-Xin Lu, ${ }^{1}$ Ze-Xian Liu, ${ }^{2}$ Min Xiao, ${ }^{1}$ Qi-Nian Wu, ${ }^{1}$ Yan-Xing Chen, ${ }^{1}$ PeiShan Hu, ${ }^{2}$ Zhan-Hong Chen, ${ }^{1}$ Yun Wang, ${ }^{1}$ Jia-Huan Lu, ${ }^{1}$ Zhao-Lei Zeng, ${ }^{1}$ Huai-Qiang Ju, ${ }^{1}$ Rui-Hua Xu. 'Sun Yat-sen University Cancer Center, State Key Laboratory of Oncology in South China, Collaborative Innovation Center for Cancer Medicine, China; ${ }^{2}$ The Third Affiliated Hospital of Sun Yat-sen University, China

\subsection{6/gutjnl-2019-IDDFabstracts.73}

Background Long non-coding RNAs (lncRNAs) are emerging as key molecules in gastric cancer(GC), yet their potential molecular mechanisms are not well understood. The aim of our study is to identify lncRNAs that are up-regulated in gastric cancer tissues and explore their function in gastric cancer metastasis.

Methods RNA sequencing of 48 paired gastric tumor and non-tumor tissues in SYSUCC was conducted. The upregulated
IncRNAs were selected and overlapped with those in TCGA database. A siRNA library was established using the top 50 lncRNAs highly expressed in GC and used to identify lncRNAs that significantly affected cell migration based on transwell assays. The expression of our focused lncRNA CRCAL-2(ColoRectal Cancer Associated LncRNA-2) was measured using quantitative reverse transcription PCR assays and lncRNA in situ hybridization. In vivo,cell-based and patientderived xenograft (PDX) models were used to further explore roles of CRCAL-2 in GC metastasis. Then, RNA pull-down, mass spectrometry analyses, western blot and RNA-binding protein immunoprecipitation (RIP) were performed to identify interaction proteins and related mechanisms of CRCAL-2.

Results LncRNA CRCAL-2, significantly overexpressed in the tumor tissues, was identified as the key regulator of GC metastasis. Upregulated CRCAL-2 in GC correlated with poor overall survival. Knockdown of CRCAL-2 significantly reduced GC cells migration, invasion, and metastasis of xenograft tumors in nude mice. Mechanistically, CRCAL-2 promoted GC metastasis by directly interacting with and stabilizing the nuclear transport protein RAN, resulting in increasement of nuclear import of beta-catenin and ultimately, activation of downstream targeted EMT(Epithelial-Mesenchymal Transition) genes. Additionally, the transcription factor YY1 could bind to the promoter of CRCAL-2 to upregulate its expression.

Conclusions Our results suggest that YY1/CRCAL2/beta-catenin axis play a crucial role in GC metastasis, and the newly identified lncRNA CRCAL-2 might be developed as a biomarker and potential therapeutic target of GC in patients.

\section{IDDF2019-ABS-0317 INCIDENCE OF HELICOBACTER PYLORI ANTIBIOTIC RESISTANCE A SINGLE CENTER CROSS-SECTIONAL STUDY}

${ }^{1}$ Zcharmaine Yumang ${ }^{*}$, ${ }^{1}$ Edgardo Bondoc, ${ }^{1} J o h n$ Arnel Pangilinan, ${ }^{2}$ Maria Celeste Cortes, ${ }^{1}$ Henedine Gae Chua, ${ }^{1}$ Ma Marylaine Dujunco, 'Kristine Joyce Cruz, ${ }^{2}$ Ma Luisa Juan, ${ }^{2}$ Michael Kim Valdez, ${ }^{2}$ Maria Katrina Morato, ${ }^{3}$ Yoshio Yamaoka, ${ }^{4}$ Takeshi Azuma. ${ }^{1}$ Institute of Digestive and Liver Diseases, St. Luke's Medical Center - Quezon City, Philippines; ${ }^{2}$ Research and Biotechnology St. Luke's Medical Center - Quezon City, Philippines; ${ }^{3}$ Oita University, Oita, Japan; ${ }^{4}$ Kobe University Graduate School of Medicine, Japan

\subsection{6/gutjin-2019-IDDFabstracts.74}

Background Helicobacter pylori infection has become increasingly resistant to traditional first-line treatment regimens because of emerging antibiotic resistance coupled with poor patient compliance with completing the treatment course. There is limited data regarding the incidence of $\mathrm{H}$. pylori treatment resistance in the Philippines. This study aims to guide physicians in deciding the choice of the treatment regimen for both treatment-naive and treatment-resistant organisms.

Methods This is a prospective, cohort study in St. Luke's Medical Center. We currently enrolled 73 patients positive for H. pylori infection based on rapid urease test, and gastroscopy, biopsy of the antrum, incisura and corpus and subsequent $H$. pylori culture and sensitivity against amoxicillin, clarithromycin, metronidazole, tetracycline, and levofloxacin were done.

Results Thirty-eight percent $(38 \%)$ or 28 out of 73 patients enrolled are $\mathrm{H}$. pylori culture positive and we were able to isolate and do sensitivity analysis in 42 samples. The incidence rate of Helicobacter treatment-resistant infection is high at $66.7 \%$ or 28 out of 42 isolates. Among the medications used, 